\title{
Design of High-Sensitivity Surface Plasmon Resonance Sensor Based on Nanostructured Thin Films for Effective Detection of DNA Hybridization
}

\section{Reza Ghayoor}

Sharif University of Technology

Soraya Zangenehzadeh

Shiraz University of Technology

Alireza Keshavarz ( $\nabla$ keshavarz@sutech.ac.ir)

Shiraz University of Technology https://orcid.org/0000-0003-3637-7005

\section{Research Article}

Keywords: Biosensor based on thin film, Coronavirus, Quasi-photonic crystal, Graphene, Surface plasmon resonance

Posted Date: March 1st, 2022

DOI: https://doi.org/10.21203/rs.3.rs-1363076/v1

License: (c) (i) This work is licensed under a Creative Commons Attribution 4.0 International License. Read Full License 


\section{Design of High-Sensitivity Surface Plasmon Resonance Sensor Based on Nanostructured Thin Films for Effective Detection of DNA Hybridization}

Reza Ghayoor ${ }^{1}$, Soraya Zangenehzadeh ${ }^{2}$, and Alireza Keshavarz ${ }^{2 *}$

${ }^{1}$ Institute for Nanoscience and Nanotechnology, Sharif University of Technology, Tehran, Iran

2 Department of Physics, Shiraz University of Technology, Shiraz, Iran;

*Corresponding author: Alireza Keshavarz, keshavarz@ sutech.ac.ir

\section{Abstract}

With the increasing ability to control infectious diseases in developed countries, there has come the realization that genetic diseases are a major cause of disability, death, and human tragedy. Recently, Coronavirus as an epidemic, has spread worldwide and the ability to identify low concentrations and the mutations of the virus can effectively reduce the spread of the disease. In this paper, A surface plasmon resonance sensor based on nanostructured thin films and graphene as a 2D material has been designed with high sensitivity and accuracy to identify DNAbased infectious diseases such as SARS-CoV-2. The effects of different structural factors, including nanolayers thickness on the sensor's performance are assessed through the transfer matrix method. The results demonstrated that the sensor with the Kretschmann configuration has ultra-high sensitivity (192.19 deg/RIU) and a high figure of merit $\left(634.68 \mathrm{RIU}^{-1}\right)$.

Keywords: Biosensor based on thin film; Coronavirus; Quasi-photonic crystal; Graphene; Surface plasmon resonance 


\section{INTRODUCTION}

The sensors based on arrays of immobilized single-stranded DNA (ss-DNA) are known as quick, sensitive, and stable methods for the detection of sequencing of the human genome and the diagnosis of genetic disease. The performance of this sensors is based on the hybridization of active sites of ss-DNA molecules(probe) with the liquid sample containing the DNA target. The detection of hybridization can be obtained through changes in the electrochemical properties or changes in the optical properties due to nonspecifically adsorbed target molecules. The physical absorption of DNA target molecules can be monitored with the surface plasmon resonance (SPR) method[1-4].

The SPR is related to the quantum of massive oscillations of free electrons in a metal-dielectric interface. The presence of a surface or interface between materials with different dielectric constants can lead to specific surface excitations, and these waves are enclosed near the interface[5-7]. The Kretschmann structure is used in the SPR sensor applications such as biochemical sensors, gas sensing, and diagnostics of medical issues. These structures include a prism, a metal, a dielectric, and a sensitive medium[8-10]. Using different materials for the metal and dielectric layers and changes in layered structures, the sensitivity of the SPR method can be improved. Graphene is one of the 2D materials that can greatly enhance localized surface plasmons due to its adjustable electrical properties[11]. Furthermore, quasi-photonic crystals as a new kind of photonic crystal have emerged[12] that are used for a variety of applications, such as waveguides[13], filters[14-16], and optical sensors[17]. Quasi-photonic crystals as the new class of stack layers give an extra degree of freedom for tailoring photonics and photonic bandgaps based on their spectral gaps in the frequency domain. The Fibonacci structure is one of the quasi-photonic crystals composed of dielectric layers[18] and is expected to improve the sensitivity of the SPR method sensitivity.

In recent years, given the increasing need of humans to control the COVID-19 pandemic, it is necessary to use a precise tool to identify and control these respiratory infection hazards. This novel 
coronavirus was first discovered through viral metagenomic analyses conducted on three bronchoalveolar-lavage specimens from patients with severe pneumonia symptoms. Both coronavirus and the common cold cause respiratory disease and using the precise diagnosis method is still challenging[19,20]. As the virus spreads out through the world, applying accurate technology to detect COVID-19 is a crucial issue. Detecting the low concentration and mutation of coronavirus in body fluid samples such as nasal mucus membrane and human blood has been tested by an available method including nasopharyngeal swab and serologic tests. Recent studies have reported errors in COVID-19 diagnostic tests. For patients with heart problems or weakened immune systems, using non-invasive and accurate technology to test their body sample is necessary, which is possible by optical sensing[21-23].

In this paper, a novel DNA hybridization SPR sensor based on the nanostructured thin films with realtime detection is proposed. The hybridization changes in the interface between the thiol-tethered DNA film and the DNA target (i.e. the human nasopharyngeal swabs include SARS-CoV-2) were monitored with a series of SPR spectra. The introduction of Fibonacci quasi-photonic crystal contributes to the improvement of detection and sensitivity in the sensor.

\section{DESIGN AND THEORETICAL MODEL}

\subsection{Proposed Structure}

Quasi-photonic crystals can be grown by juxtaposing the two building blocks $H$ and $L$ where $H$ and $L$ correspond to the high and low refractive indexes materials with refractive indices $n_{H}$ and $n_{L}$, respectively. The Fibonacci sequence can be used in the quasi-photonic crystals. These structures can be produced by irregular repeating the substitution rule $H \rightarrow H L$ and $L \rightarrow H H$.

The first few generations $S_{\mathrm{m}}$ in the Fibonacci sequence are as follows: $S_{0}=\{H\}, S_{1}=\{L\}$, $S_{2}=\{H L\}, S_{3}=\{L H L\}$ and $S_{4}=\{H L L H L\}[24]$. 
The SPR angle and the minimum reflection energy were studied for monitoring the SARS-CoV-2. The configuration and the design of the proposed sensor are shown in Figure 1. The gold metallic nanolayer (plasmonic metal) is coated on an SF10 prism, and then the Fibonacci quasi-photonic crystal is composed of graphene (as a $2 \mathrm{D}$ material) and $\mathrm{Bi}_{4} \mathrm{Ti}_{3} \mathrm{O}_{12}$ thin films are deposited, respectively. We developed the Fibonacci quasi-photonic structure functionalized with the excellent receptor of SARSCoV-2 as one of the applications of the thiol tethered DNA. Here, the antigen is the SARS-CoV-2 virus is taken as the sensing medium which is in serum/nasopharyngeal swab samples.

\subsection{Computational Method}

The analysis of reflectivity in multilayer scheme sensors is performed by the transfer matrix method that is an accurate and efficient method to calculate the reflection coefficient. This method is used to link the magnetic and the electric fields tangential components of the first and outer layers.

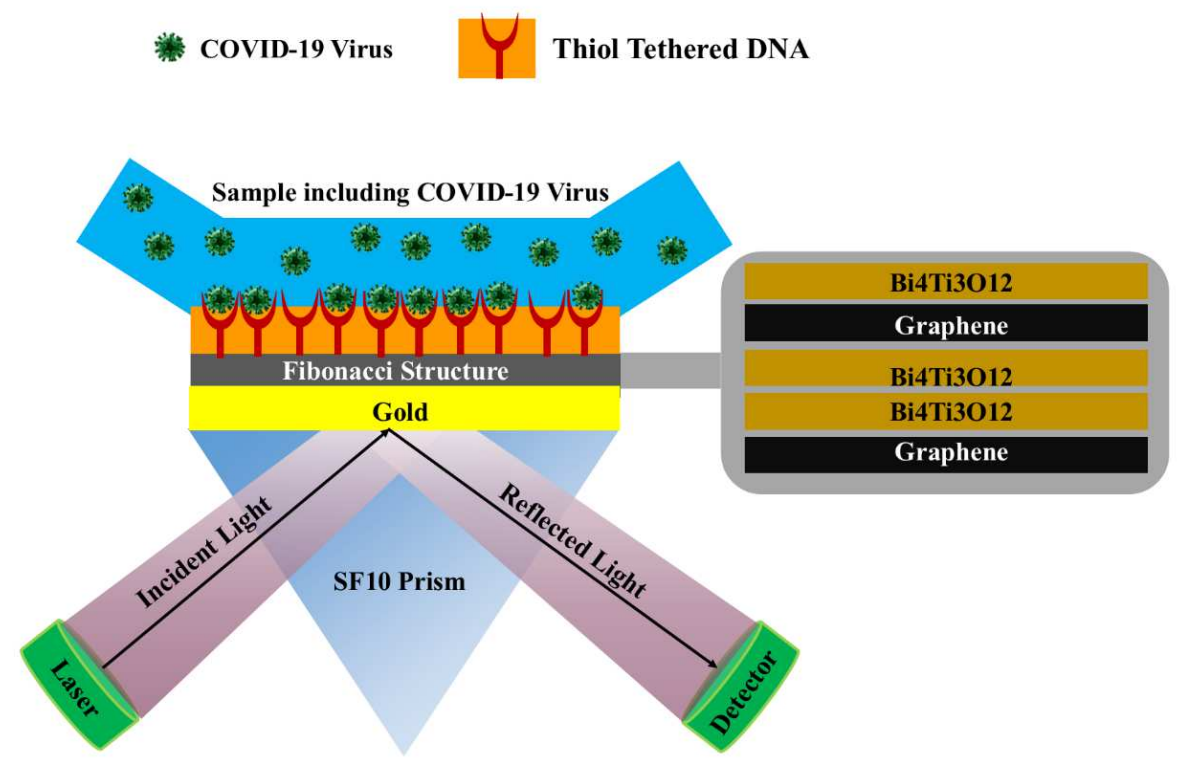

Figure 1. Schematic of the proposed SPR sensor configuration including the gold nanolayer and nanostructured thin films. 
Here, the incident light propagation in the sensor layers is investigated by the transfer matrix and Fresnel's equations. The interface matrix will be considered as $M$ which can link the electric field of the layer $i$ to the layer $i+1[25]$ :

$$
M_{i}=\left[\begin{array}{cc}
\cos \left(b_{i}\right) & -i \sin \left(b_{i}\right) / q_{i} \\
-i q_{i} \sin \left(b_{i}\right) & \cos \left(b_{i}\right)
\end{array}\right]
$$

Where the admittance $b_{i}$ and the phase shift $\boldsymbol{q}_{i}$ parameters define as follows:

$$
\begin{gathered}
b_{i}=\frac{2 \pi d_{i}}{\lambda \sqrt{n_{i}-\left(n_{1} \sin \theta\right)^{2}}} \\
q_{i}=\frac{\sqrt{n_{i}-\left(n_{1} \sin \theta\right)^{2}}}{n^{2}{ }_{i}}
\end{gathered}
$$

The $n_{i}$ and $d_{i}$ are the refractive index and thickness of layer $i^{\text {th }}$ respectively, $\lambda$ is the incident light wavelength, $\theta$ is the angle of the incident light and $n_{l}$ is the refractive index of the prism glass. For the sensor structure in Figure 1, the entire matrix $U$ is obtained by the following equation:

$$
U=\prod_{n=2}^{N-1} M_{n}
$$

The total reflection for the p-polarized $(\mathrm{TM})$ incident light wave $\left(\mathrm{r}_{\mathrm{p}}\right)$ can be finally obtained from the elements of the matrix $U$ as[25]:

$$
r_{p}=\frac{\left(U(1,1)+U(1,2) q_{i}\right) q_{1}-\left(U(2,1)+U(2,2) q_{i}\right)}{\left(U(1,1)+U(1,2) q_{i}\right) q_{1}+\left(U(2,1)+U(2,2) q_{i}\right)}
$$

The reflectivity for defined multilayer configuration can be given by the following equation:

$$
R=\left|r_{p}\right|^{2}
$$


The principle of the SPR sensors is based on SPs interacting with the incident p-polarised light. Because the surface plasmons are not directly excited at the metallic area, the phase-matching conditions to provide the necessary momentum to excite surface plasmons are achieved by the incident of the p-polarised light on the glass prism [7].

The crucial parameters for an SPR sensor can be characterized by mainly parameters i.e., sensitivity, and figure of merit (FoM). These parameters should be high as possible for a good SPR-based sensor and calculated by resonance angle and reflectance curve changes in different incident angles. The sensitivity $(S)$ parameter is defined as a change in the resonance angle $\left(\triangle \theta_{S P R}\right)$ to change in the refractive index of the sensing medium $\left(\Delta n_{s}\right)$ and shows the ability of the SPR-based sensor to identify the change in refractive index (RI) of the sensing medium and is given by[26]:

$$
S=\frac{\Delta \theta_{S P R}}{\Delta n_{s}}
$$

The FoM is an intelligent and important scale that is defined as the resonance angle shift due to change in sensing medium divided by the FWHM:

$$
F O M=\frac{\Delta \theta_{S P R} / \Delta n_{s}}{F W H M \cdot R_{\min }}=\frac{S}{F W H M \cdot R_{\min }}
$$

Where $F W H M$ is full width at half maximum of the reflectance curve.

\section{RESULTS AND DISCUSSION}

This configuration is stimulated by a $\mathrm{He}-\mathrm{Ne}$ laser as a light excitation source with wavelength of $632.8 \mathrm{~nm}$. The refractive index of the bismuth titanate thin film in the proposed structure is considered $n_{b}=2.6477\left(\mathrm{Bi}_{4} \mathrm{Ti}_{3} \mathrm{O}_{12}\right)$ [27] with thickness of $d_{B i_{4} T_{3} O_{12}}$ which acts as the material with higher refractive index $\left(n_{H}\right)$ in the Fibonacci structure. On the other hand, the refractive index of 
graphene varies according to its chemical potential (its conductivity) which the relationship is defined as follows [28]:

$$
n_{g}(\omega)=\sqrt{\frac{1+\sigma_{g}}{i \omega \varepsilon_{0}}}
$$

Also, the isotropic surface conductivity $\sigma_{g}$ of graphene can be expressed as[29]:

$$
\sigma_{g}=\frac{i e^{2} \mu_{c}}{\pi \hbar^{2}\left(\omega+i \tau^{-1}\right)}
$$

Where $\hbar$ is the reduced Planck constant, $\boldsymbol{e}$ is the electron charge, $\mu_{c}$ is the chemical potential of graphene, and $\tau$ is the relaxation time of charge carriers. The value of this refractive index is always less than the refractive index of bismuth titanate (even with the change in chemical potential). Therefore, it appears as a material with a lower refractive index $\left(n_{L}\right)$ in the Fibonacci structure.

The thickness of graphene thin film is defined as $d_{g}$ that was selected according to the number of graphene layers $(L): d_{g}=L \times 0.34(\mathrm{~nm})$. The glass prism in the proposed sensor is selected SF10 and its refractive index is dependent on the source wavelength and is given as follows[30]:

$$
n(\lambda)=\left(\frac{1.62153902 \lambda^{2}}{\lambda^{2}-0.0122241457}+\frac{0.256287842 \lambda^{2}}{\lambda^{2}-0.0595736775}+\frac{1.64447552 \lambda^{2}}{\lambda^{2}-147.468793}+1\right)^{\frac{1}{2}}
$$

The refractive index of thiol tethered DNA has also been determined 1.35 according to the experimental results at wavelength of $632.8 \mathrm{~nm}[31]$. The resonance angle of the SPR curve changes with the sample attachment[32]. The effect of the graphene, $\mathrm{Bi}_{4} \mathrm{Ti}_{3} \mathrm{O}_{12}$, and metal nanolayers thickness, and also graphene chemical potential on the SPR curve and performance parameters of the sensor was analyzed and compared with other novel structures.

The graphene chemical potential is one of the most important factors which can directly be related to the graphene refractive index and thus reflectance coefficient. Figure 2(a)-(d) shows the variation of 
the reflectance with incident angle curve at different values of $\mu_{c}$. To study the changes in chemical potential, the number of graphene layers and the thickness of bismuth titanate were considered fix, and the thickness of gold thin film also varied from $40 \mathrm{~nm}$ to $70 \mathrm{~nm}$.

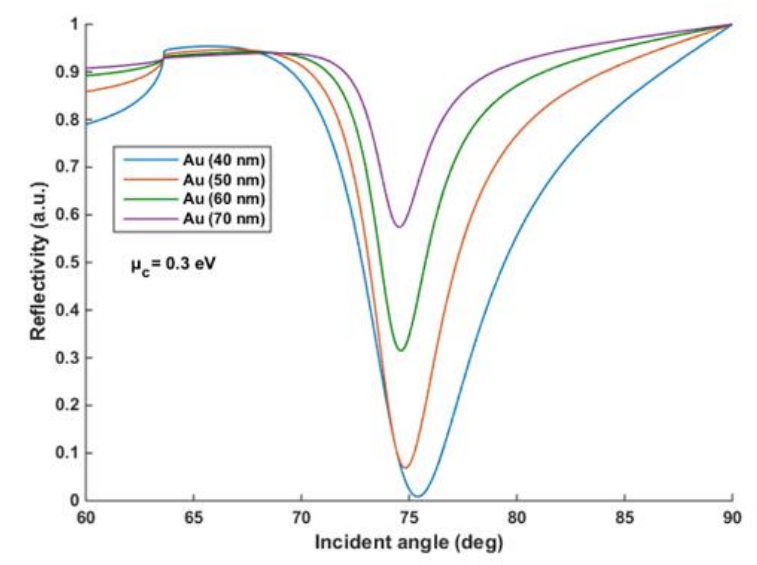

(a)

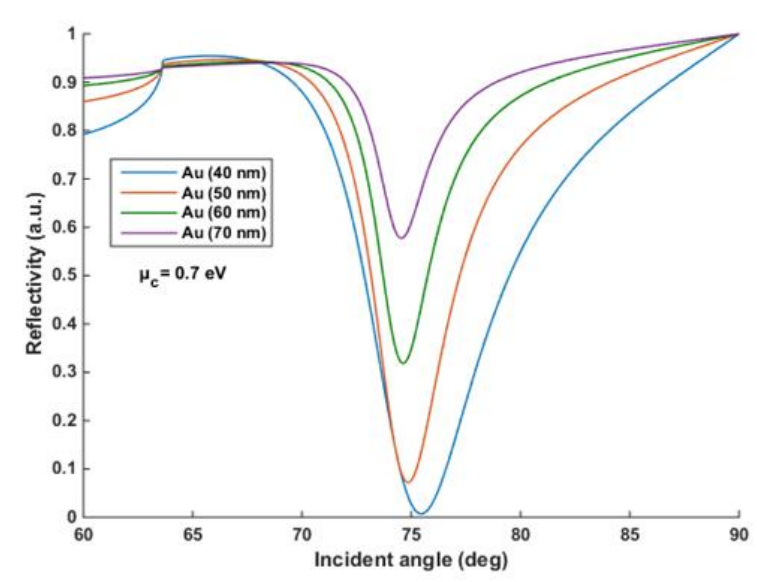

(c)

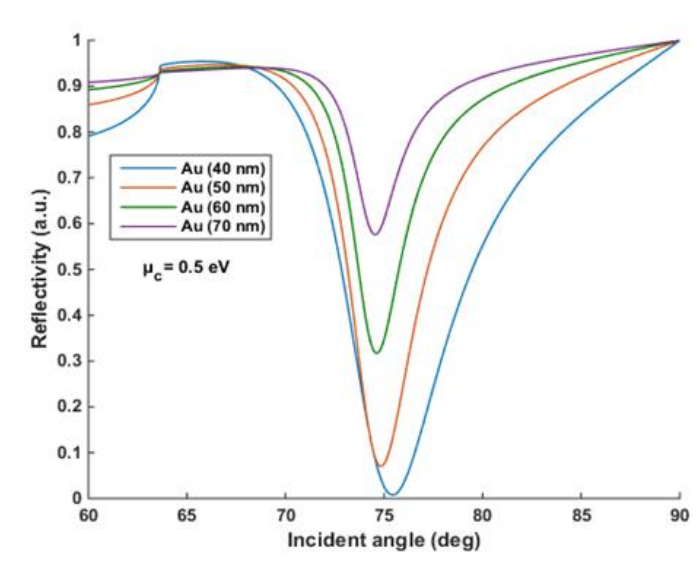

(b)

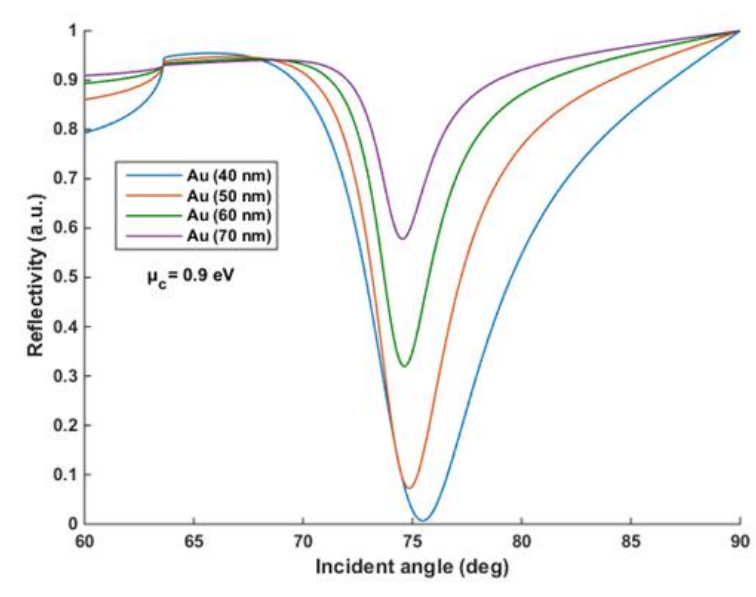

(d)

Figure 2. The reflectivity in SPR curve as a function of the graphene chemical potential with various thickness of the gold thin film (a) $\mu_{c}=0.3 \mathrm{eV}$ (b) $\mu_{c}=0.5 \mathrm{eV}$ (c) $\mu_{c}=0.7 \mathrm{eV}$ (d) $\mu_{c}=0.9 \mathrm{eV}$; with $d_{g}=4 \times 0.34 \mathrm{~nm}$ and $d_{B_{4} T_{3} \mathrm{O}_{12}}=19.5 \mathrm{~nm}$.

As shown in figure 2(a)-(d), it is found that the resonance angle remains almost constant, and reflectance minima also increase from 0.006 (a.u.) to 0.570 (a.u.) as the chemical potential changes from $0.3 \mathrm{eV}$ to $0.9 \mathrm{eV}$. Therefore, increasing the chemical potential can increase the reflectance and decrease absorption. Table 1 provides concluding information on figure 2 . 
TABLE 1. Conclusion information from figure 2.

\begin{tabular}{cccccc}
\hline $\begin{array}{c}\text { Chemical } \\
\text { potential }(\mathrm{eV})\end{array}$ & $\begin{array}{c}\text { Au thickness } \\
(\mathrm{nm})\end{array}$ & $\begin{array}{c}\text { Minimum } \\
\text { reflectivity }\end{array}$ & $\begin{array}{c}\text { Sensitivity } \\
(\mathrm{deg} / \mathrm{RIU})\end{array}$ & $\begin{array}{c}\text { FWHM } \\
(\mathrm{deg})\end{array}$ & $\begin{array}{c}\text { FoM } \\
(1 / \mathrm{RIU})\end{array}$ \\
\hline \multirow{3}{*}{0.3} & 40 & 0.007 & 204.20 & 6.66 & 396.68 \\
& 50 & 0.007 & 204.20 & 6.67 & 429.48 \\
& 60 & 0.006 & 204.20 & 6.67 & 454.52 \\
& 70 & 0.006 & 204.20 & 6.68 & 474.15 \\
\hline & 40 & 0.070 & 192.19 & 4.39 & 618.66 \\
& 50 & 0.072 & 192.19 & 4.39 & 607.02 \\
& 60 & 0.072 & 186.18 & 4.39 & 580.23 \\
& 70 & 0.073 & 192.19 & 4.40 & 593.55 \\
\hline & 40 & 0.317 & 186.18 & 3.48 & 168.30 \\
& 50 & 0.319 & 186.18 & 3.48 & 167.35 \\
& 60 & 0.320 & 186.18 & 3.48 & 166.68 \\
0.7 & 70 & 0.321 & 186.18 & 3.48 & 166.19 \\
\hline & 40 & 0.570 & 186.18 & 3.20 & 100.66 \\
& 50 & 0.570 & 186.18 & 3.20 & 100.41 \\
& 60 & 0.570 & 186.18 & 3.21 & 100.23 \\
& 70 & 0.570 & 186.18 & 3.21 & 100.11 \\
\hline
\end{tabular}

The increasing chemical potential of graphene leads to a decrease in the sensitivity of the proposed structure. In addition, FWHM decreases from 6.68 (deg) to 3.20 (deg) which shows an increase in the sensor resolution. The best value for the FoM parameter is 618.66 (1/RIU), which is obtained at a chemical potential of $0.5 \mathrm{eV}$ and a thickness of $40 \mathrm{~nm}$ gold thin film.

To optimize the number of graphene layers, the changes of minimum reflectivity are plotted with various thicknesses of the gold thin film (i.e., $40 \mathrm{~nm}, 50 \mathrm{~nm}, 60 \mathrm{~nm}, 70 \mathrm{~nm}$ ) at a constant thickness of bismuth titanate. Figure 3(a)-(d) shows the reflectance versus incident angle curve for the number of different layers of graphene in the proposed configuration. When the number of graphene layers was fixed, the resonance angle shifts to smaller angles as the gold thickness increases. The lowest reflection is obtained for single-layer graphene at a gold thickness of $40 \mathrm{~nm}$. As shown in SPR curves in figure 3(a)-(d), the reflectance minima decrease, and therefore the absorption increases with an increasing number of graphene layers. 


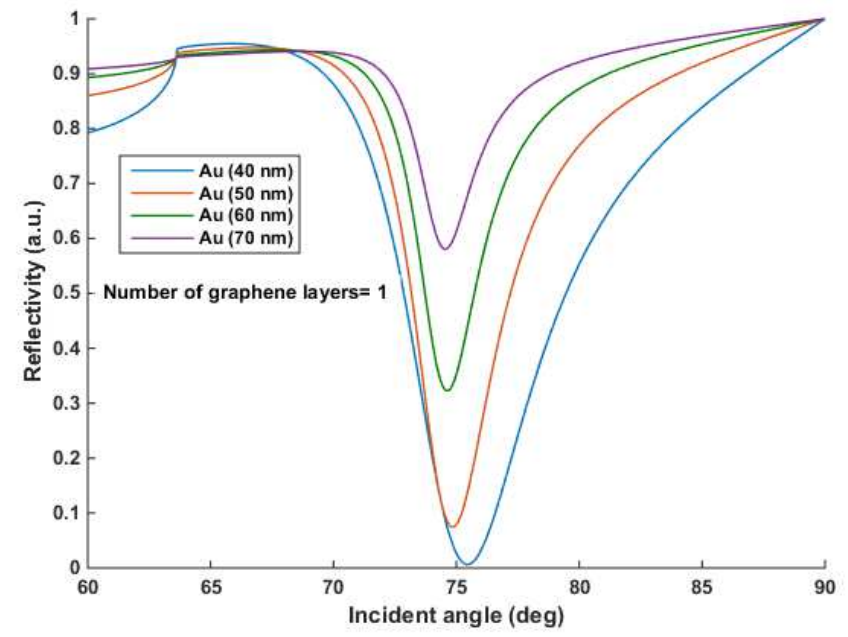

(a)

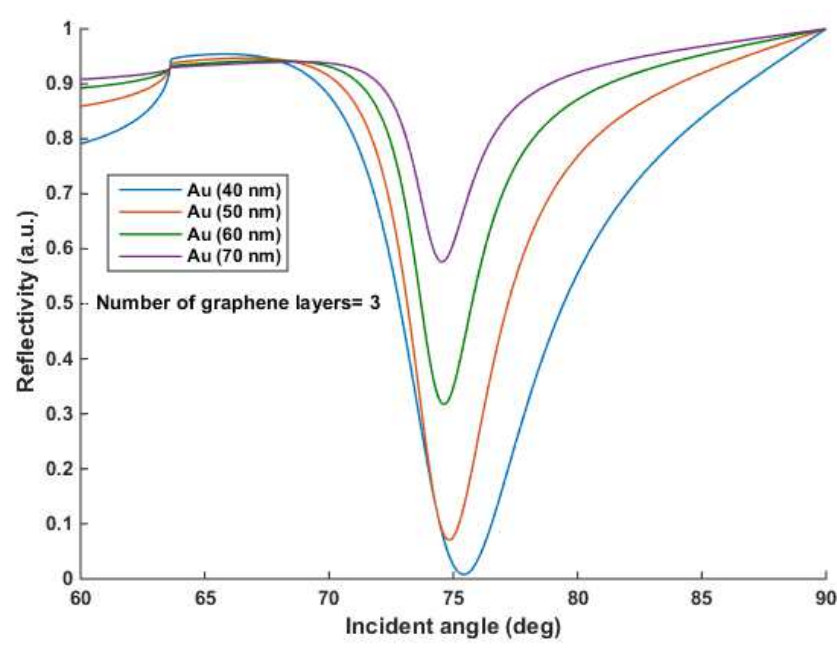

(c)

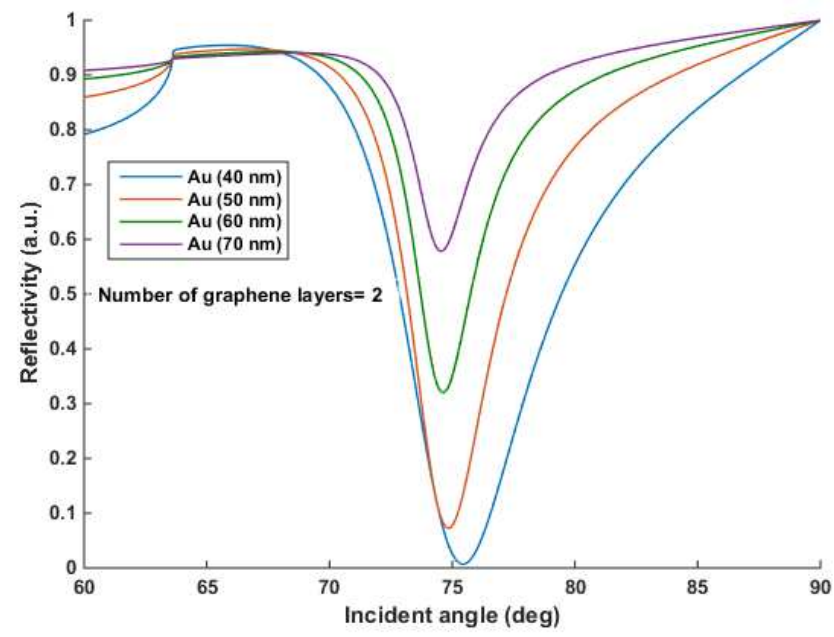

(b)

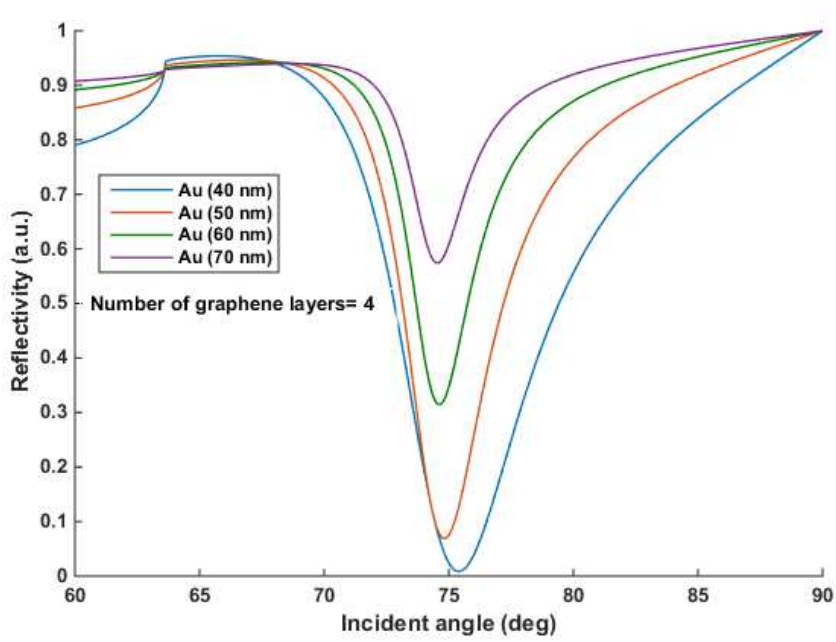

(d)

Figure 3. The reflectivity in the SPR curve as a function of the number of graphene layers $(L)$ with various thicknesses of the gold thin film (a) $L=1$ (b) $L=2$ (c) $L=3$ (d) $L=4$; with $\mu_{c}=0.3 \mathrm{eV}$ and $d_{B i_{4} T_{3} \mathrm{O}_{12}}=19.5 \mathrm{~nm}$. The performance parameters of the sensor for the number of graphene layers are listed in Table 2. As shown in Table 2, with the increasing number of graphene layers, the minimum reflectivity has occurred in larger quantities and FWHM is also reduced. As the number of graphene layers increases to $L=3$, the sensitivity first decreases and then remains constant. It seems that the few-layer graphene has a high energy barrier and high absorption efficiency. In an SPR sensor, the resolution depends on the FWHM of the reflectivity curve. The high resolution and sensing accuracy are the results of lower FWHM. As can be seen in Table 2, with the increasing number of graphene layers, the FWHM parameter has decreased from 6.67 ( $\mathrm{deg}$ ) (for $L=1$ ) to 3.20 (deg) (for $L=4$ ). 
TABLE 2. Conclusion information from figure 3.

\begin{tabular}{cccccc}
\hline $\begin{array}{c}\text { Number of } \\
\text { graphene layers }\end{array}$ & $\begin{array}{c}\text { Au thickness } \\
(\mathrm{nm})\end{array}$ & $\begin{array}{c}\text { Minimum } \\
\text { reflectivity }\end{array}$ & $\begin{array}{c}\text { Sensitivity } \\
(\mathrm{deg} / \mathrm{RIU})\end{array}$ & $\begin{array}{c}\text { FWHM } \\
(\mathrm{deg})\end{array}$ & $\begin{array}{c}\text { FoM } \\
(1 / \mathrm{RIU})\end{array}$ \\
\hline \multirow{2}{*}{$\mathrm{L}=1$} & 40 & 0.006 & 198.19 & 6.64 & 476.16 \\
& 50 & 0.006 & 204.20 & 6.65 & 441.28 \\
& 60 & 0.007 & 204.20 & 6.66 & 396.68 \\
& 70 & 0.008 & 204.20 & 6.67 & 359.83 \\
\hline & 40 & 0.074 & 192.19 & 4.38 & 588.27 \\
& 50 & 0.072 & 192.19 & 4.38 & 603.20 \\
& 60 & 0.070 & 192.19 & 4.39 & 618.66 \\
$\mathrm{~L}=2$ & 70 & 0.068 & 192.19 & 4.39 & 634.68 \\
\hline & 40 & 0.322 & 186.18 & 3.48 & 165.75 \\
& 50 & 0.320 & 186.18 & 3.48 & 167.02 \\
& 60 & 0.317 & 186.18 & 3.48 & 168.30 \\
$\mathrm{~L}=4$ & 70 & 0.314 & 186.18 & 3.48 & 169.61 \\
\hline & 40 & 0.580 & 186.18 & 3.20 & 100.01 \\
& 50 & 0.578 & 186.18 & 3.20 & 100.33 \\
& 60 & 0.576 & 186.18 & 3.20 & 100.66 \\
& 70 & 0.574 & 186.18 & 3.21 & 100.99 \\
\hline
\end{tabular}

Similarly, the reflectance curve was plotted for different thicknesses of $\mathrm{Bi}_{4} \mathrm{Ti}_{3} \mathrm{O}_{12}$. The resonance angles are shifted to higher angles as the $\mathrm{Bi}_{4} \mathrm{Ti}_{3} \mathrm{O}_{12}$ thickness has increased from $6.5 \mathrm{~nm}$ to $26 \mathrm{~nm}$ and reflectance minima increase from 0.008 (a.u.) to 0.585 (a.u.) as shown in figure $4(a)-(d)$.

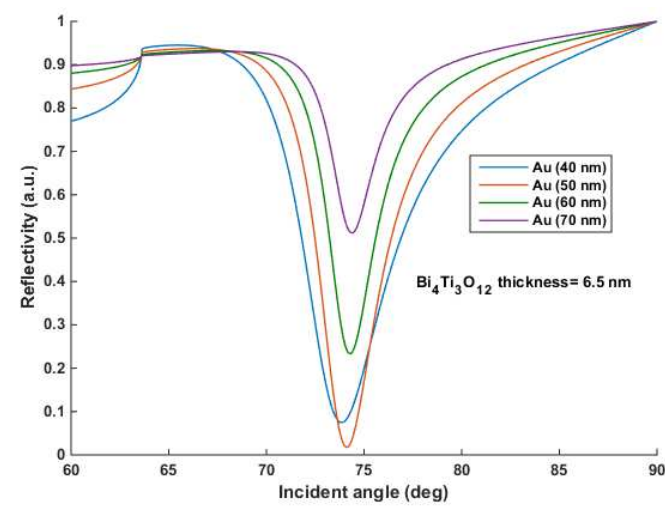

(a)

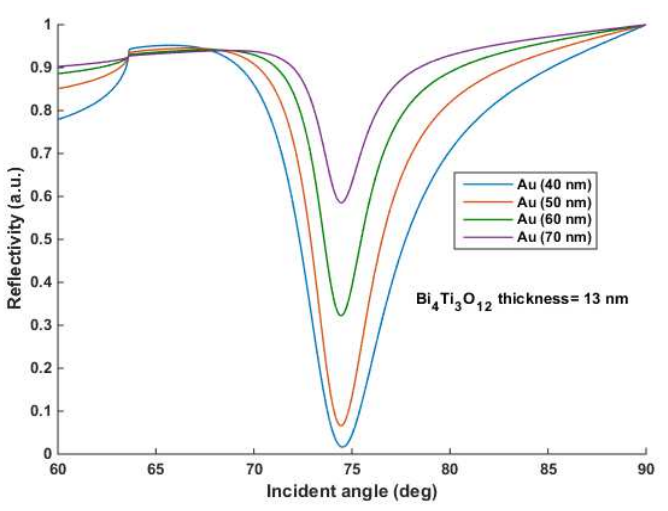

(b) 


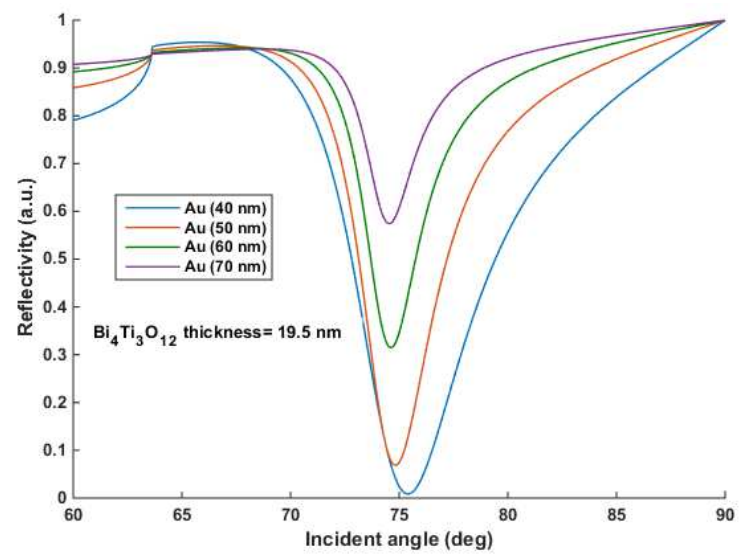

(c)

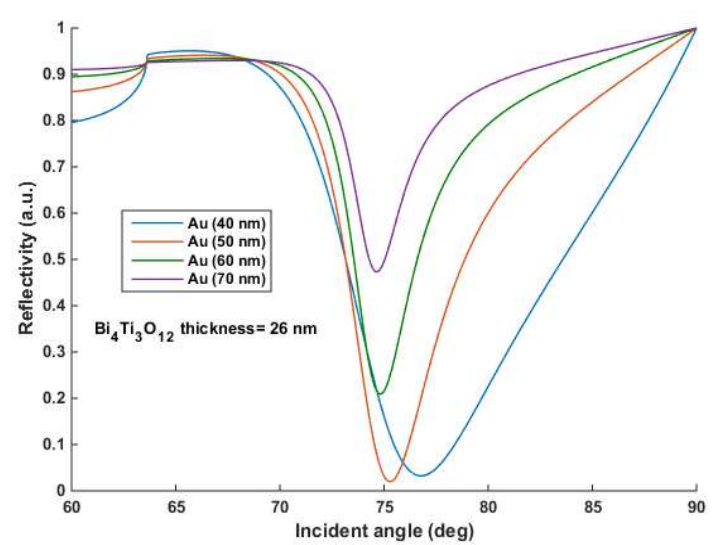

(d)

Figure 4. The reflectivity in SPR curve as a function of the $\mathrm{Bi}_{4} \mathrm{Ti}_{3} \mathrm{O}_{12}$ thicknesses with various thicknesses of the gold thin film (a) $\mathrm{Bi}_{4} \mathrm{Ti}_{3} \mathrm{O}_{12}$ thicknesses= $6.5 \mathrm{~nm}$ (b) $\mathrm{Bi}_{4} \mathrm{Ti}_{3} \mathrm{O}_{12}$ thicknesses $=13 \mathrm{~nm}$ (c) $\mathrm{Bi}_{4} \mathrm{Ti}_{3} \mathrm{O}_{12}$ thicknesses $=19.5 \mathrm{~nm}$ (d) $\mathrm{Bi}_{4} \mathrm{Ti}_{3} \mathrm{O}_{12}$ thicknesses $=26 \mathrm{~nm}$; with values of $\mu_{c}=0.3 \mathrm{eV}$ and $d_{g}=4 \times 0.34 \mathrm{~nm}$.

The $\mathrm{Bi}_{4} \mathrm{Ti}_{3} \mathrm{O}_{12}$ thickness analysis showed that for the thickness of $6.5 \mathrm{~nm}$ in this configuration, the sensitivity will be 258.25 (deg/RIU). A comparison between the performance parameters of the sensor with different thicknesses of $\mathrm{Bi}_{4} \mathrm{Ti}_{3} \mathrm{O}_{12}$ is listed in Table 3. As Table 3 shows, at a constant thickness of bismuth titanate, the sensitivity has been greatly improved with increasing thickness of gold thin film but the FWHM has also increased which is undesirable. In addition, the results show that the sensitivity in this configuration is more affected by the gold thickness changes while the changes of sensitivity was less than the changes of gold thickness (in case of investigation of the chemical potential of graphene and number of graphene layers). From the comparison between the results of graphene layer thickness changes and changes of $\mathrm{Bi}_{4} \mathrm{Ti}_{3} \mathrm{O}_{12}$ layer thickness, it can be seen that the performance of the sensor strongly depends on the geometry of the structure because the number of layers with lower refractive index in Fibonacci structure is less than the number of layers with the higher refractive index. 
TABLE 3. Conclusion information from Figure 4.

\begin{tabular}{cccccc}
\hline $\begin{array}{c}\mathrm{Bi}_{4} \mathrm{Ti}_{3} \mathrm{O}_{12} \\
\text { thickness }(\mathrm{nm})\end{array}$ & $\begin{array}{c}\text { Au thickness } \\
(\mathrm{nm})\end{array}$ & $\begin{array}{c}\text { Minimum } \\
\text { reflectivity }\end{array}$ & $\begin{array}{c}\text { Sensitivity } \\
(\mathrm{deg} / \mathrm{RIU})\end{array}$ & $\begin{array}{c}\text { FWHM } \\
(\mathrm{deg})\end{array}$ & $\begin{array}{c}\text { FoM } \\
(1 / \mathrm{RIU})\end{array}$ \\
\hline 6.5 & 40 & 0.074 & 162.16 & 5.54 & 392.18 \\
& 50 & 0.016 & 180.18 & 5.51 & 198.66 \\
& 60 & 0.008 & 204.20 & 6.67 & 359.83 \\
& 70 & 0.032 & 258.25 & 10.73 & 744.09 \\
\hline 13 & 40 & 0.017 & 174.17 & 3.98 & 247.94 \\
& 50 & 0.066 & 180.18 & 3.93 & 691.02 \\
& 60 & 0.068 & 192.19 & 4.39 & 634.68 \\
& 70 & 0.019 & 210.21 & 5.85 & 179.63 \\
\hline 19.5 & 40 & 0.023 & 174.17 & 3.30 & 225.64 \\
& 50 & 0.322 & 180.18 & 3.27 & 170.82 \\
& 60 & 0.314 & 186.18 & 3.48 & 169.61 \\
& 70 & 0.020 & 192.19 & 4.11 & 223.04 \\
\hline 26 & 40 & 0.511 & 180.18 & 3.12 & 112.80 \\
& 50 & 0.585 & 186.18 & 3.09 & 102.81 \\
& 60 & 0.574 & 186.18 & 3.21 & 100.99 \\
& 70 & 0.473 & 186.18 & 3.55 & 110.59 \\
\hline
\end{tabular}

The thickness of the layers was optimized in a layer-wise procedure to achieve maximum sensor performance. Initially, the effect of the thickness of each layer on the reflectivity as a function of incident angle and sensitivity was investigated and the thickness of the other layers was kept constant. After optimizing the thickness of each layer, this process was repeated for the other layers. The goal was to get the lowest $R_{\min }$ (minimum value of the reflectivity curve) at the same time the most sensitivity. This process was performed for gold, graphene and bismuth titanate layers. Figure 5 shows the 3D diagrams depicting the sensitivity as a function of the chemical potential of graphene, the number of graphene layers, and bismuth titanate thickness, simultaneously. From Figure 5 (a), the highest value of sensitivity seemed to be attainable with lower thicknesses of bismuth titanate and a 


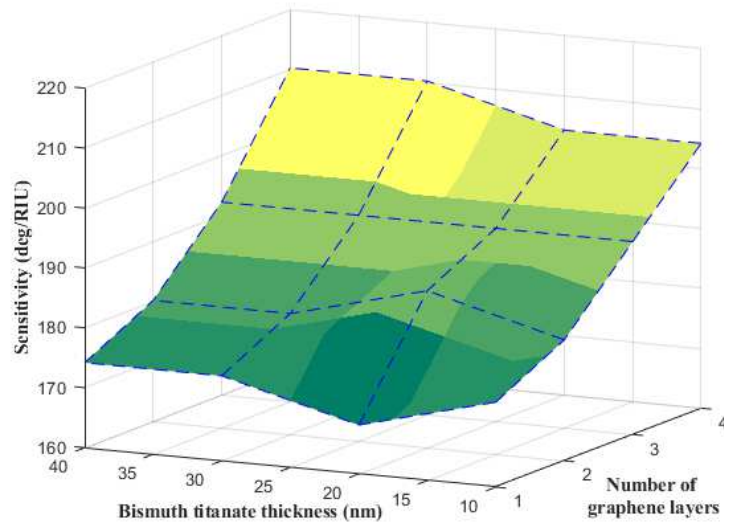

(a)

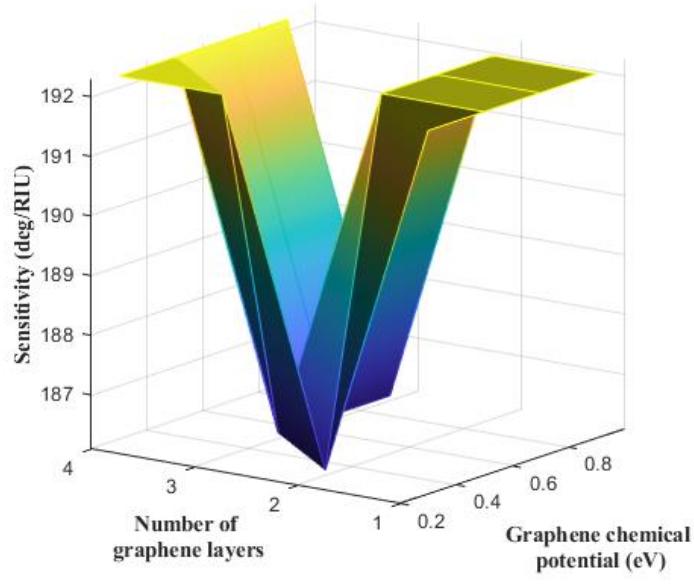

(b)

Figure 5. The variation of the sensitivity as a function of the chemical potential of graphene, number of graphene layers, and bismuth titanate thickness with $50 \mathrm{~nm}$ gold thin film (a) The effect of graphene and bismuth titanate thickness changes on sensitivity; (b) The effect of graphene chemical potential and bismuth titanate thickness changes on sensitivity.

large number of graphene layers. According to the above results, here, the optimal thickness of the gold thin film was considered $50 \mathrm{~nm}$. Although the sensitivity can increase in a certain range but according to the results, increasing sensitivity can be accompanied with a decrease in the FoM parameter. Therefore, a compromise will be made to obtain the optimal value of sensitivity and the FoM parameter at the same time. The optimum thickness for bismuth titanate and the number of graphene layers should be $19.5 \mathrm{~nm}$ and $\mathrm{L}=4$, respectively, to meet this demand. Figure 5 (b) also shows the sensitivity changes for the chemical potential of graphene (graphene refractive index) at different thicknesses of graphene. It is cleared the sensitivity at $\mathrm{L}=2$ suddenly decreases for all values of chemical potential. The high real part refractive index of graphene layers and the high dielectric constant of $\mathrm{Bi}_{4} \mathrm{Ti}_{3} \mathrm{O}_{12}$ can enhance the evanescent field at the metal interface and can serve as well as the absorption medium. In the Fibonacci structure, the refractive index effect of the bismuth titanate layer can be more effective because in this structure, the number of layers with a higher refractive index (bismuth titanate) is more than the number of layers with a lower refractive index (graphene). 
Figure 6 shows the reflectance curves for analyte refractive index changes from 1.33 to 1.35 in steps of 0.005 . Any change in the refractive index of the sensing medium causes a change in the resonance angle of the surface plasmon, and consequently the sensor sensitivity changes. After the discussions described above, the configuration of SF10 prism-gold thin film-Fibonacci structure-thiol tethered DNA-sensing medium was optimized and the reflection curve is plotted. The number of graphene layers and $\mathrm{Bi}_{4} \mathrm{Ti}_{3} \mathrm{O}_{12}$ thickness determined $L=4$ and $19.5 \mathrm{~nm}$, respectively. In addition, the optimal refractive index of graphene was calculated by chemical potential and found as a material with a lower refractive index in the Fibonacci structure. In Figure 6, the reflectance minima of the sensors are reduced with the increment of sensing medium refractive index while the SPR curve is shifting from the left to the right direction.

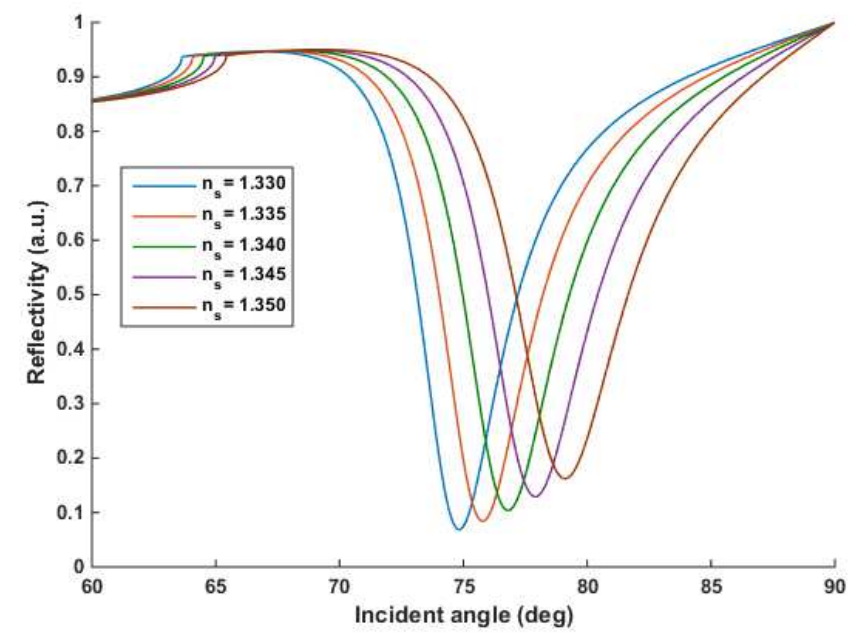

Figure 6. Variation of reflectivity in terms of the incident angle of light for analyte coronavirus (as sensing medium) ranging from 1.33 to 1.35 with an interval of 0.005 for SPR sensor with the configuration of SF10 prism-gold layer-Fibonacci structure- thiol tethered DNA-sensing medium.

As Figure 6 shows, the resonance angles of the SPR curves for SF10/Au (50 nm)/ Fibonacci structure (including graphene and $\mathrm{Bi}_{4} \mathrm{Ti}_{3} \mathrm{O}_{12}$ layers)/ are obtained at $74.83 \circ, 75.79 \circ, 76.78^{\circ}, 77.89^{\circ}$, and $79.09^{\circ}$ for analyte coronavirus of $1.330,1.335,1.340,1.345$, and 1.350 , respectively. With a slight increase of refractive index, there is a noticeable variation in sensitivity as high as $\mathbf{2 6 4 . 2 6}$ (deg/RIU) which is related to the refractive index of 1.350 . 
Finally, the performance parameters of the proposed configuration with other structures in the field of detection are compared. The sensitivity, FWHM and FoM were taken into account for comparison. However, the sensitivity is the most important performance parameter under consideration, FWHM and FoM are also crucial for sensing accuracy. The findings of this comparison are presented in Table 4. It is clear that the proposed design shows higher sensitivity than other structures. The proposed structure simultaneously presented high sensitivity and lower FWHM while some designs have relatively good sensitivity but they suffer from higher FWHM.

TABLE 4. Sensing performance comparison with other structures.

\begin{tabular}{cccc}
\hline Sensor configuration & $\begin{array}{c}\text { Sensitivity } \\
(\mathrm{deg} / \mathrm{RIU})\end{array}$ & FWHM (deg) & FoM (RIU $\left.{ }^{-1}\right)$ \\
\hline This work (proposed structure) & $\mathbf{1 9 2 . 1 9}$ & $\mathbf{4 . 3 9}$ & $\mathbf{6 3 4 . 6 8}$ \\
\hline $\mathrm{Ag}-\mathrm{Si}_{-} \mathrm{BaTiO}_{3}[33]$ & 130.30 & 11.86 & 692.28 \\
\hline $\begin{array}{c}\text { Gold nanosheet is functionalized } \\
\text { with SARS-CoV-2 } \\
\text { spike (S) protein antibody[34] }\end{array}$ & 111.11 & Not reported & Not reported \\
\hline $\begin{array}{c}\text { Mirrored bilayer of Au-MoS }{ }_{2}- \\
\text { Graphene[35] }\end{array}$ & 75.2 & 17 & 44.23 \\
\hline $\begin{array}{c}\mathrm{TiO}_{2}-\mathrm{SiO}_{2}-\mathrm{Ag}-\mathrm{MoS}_{2}- \\
\text { Graphene[36] }\end{array}$ & 98 & Not reported & Not reported \\
\hline Au-Graphene [37] & 53.71 & 5 & 85.79 \\
\hline
\end{tabular}

\section{CONCLUSION}

In this paper, we designed an SPR sensor based on a novel configuration to detect and study DNA hybridization with high sensitivity and accuracy. The optimal configuration for this sensor was a Fibonacci structure composed of nanostructured thin films and graphene as a 2D material with localized surface plasmons properties. The effect of nanofilms thickness such as the number of graphene layers and $\mathrm{Bi}_{4} \mathrm{Ti}_{3} \mathrm{O}_{12}$ thickness on sensor performance was assessed. This assessment yielded a high sensitivity value of 192.19 (deg/RIU). Given such strong sensing performance, the proposed design can be used in a lot of high sensitivity applications such as biomedical that detects low concentrations of DNA and RNA-based viruses and helps to arrest the further spread of infectious 
diseases.

\section{Funding}

This research received no specific grant from any funding agency in the public, commercial, or notfor-profit sectors.

\section{Competing interests}

The authors have no relevant financial or non-financial interests to disclose.

\section{Availability of data and material}

The data that support the findings of this study are available from the corresponding author upon reasonable request.

\section{Authors' contributions}

We confirm that the manuscript has been read and approved by all named authors and that there are no other persons who satisfied the criteria for authorship but are not listed. We further confirm that the order of authors listed in the manuscript has been approved by all of us.

\section{Ethics approval and consent to participate}

Not applicable

\section{Consent to publication}

Not applicable 


\section{REFERdENCES}

[1] Kim J, Campbell A S, de Ávila B E F, and Wang J 2019 Wearable biosensors for healthcare monitoring Nat. Biotechnol. 37 389-406.

[2] Kamal Eddin F B and Fen Y W 2020 The principle of nanomaterials based surface plasmon resonance biosensors and its potential for dopamine detection Molecules 252769.

[3] Pitruzzello G and Krauss T F 2018 Photonic crystal resonances for sensing and imaging J. Opt. 20073004.

[4] Sabu C, Henna T K, Raphey V R, Nivitha K P, and Pramod K 2019 Advanced biosensors for glucose and insulin Biosens. Bioelectron. 141111201.

[5] Schneider, T., Jahr, N., Jatschka, J., Csaki, A., Stranik, O and Fritzsche, W. 2013 Localized surface plasmon resonance (LSPR) study of DNA hybridization at single nanoparticle transducers Journal of nanoparticle research 15 1-10.

[6] Patel S K, Parmar J, Kosta Y P, Ladumor M, Zakaria R, Nguyen T K, and Dhasarathan V 2020 Design of graphene metasurface based sensitive infrared biosensor Sensors and Actuators A Phys. 301111767.

[7] Kumar A, Yadav A K, Kushwaha A S, and Srivastava S K 2020 A comparative study among WS2, MoS2 and graphene based surface plasmon resonance (SPR) sensor Sensors and Actuators Reports 2100015.

[8] Shibayama J, Mitsutake K, Yamauchi J, and Nakano H 2021 Kretschmann-and Ottotype surface plasmon resonance waveguide sensors in the terahertz regime MICROW OPT TECHN LET. 63 103-106.

[9] Taniselass S, Arshad M M and Gopinath S C 2019 Graphene-based electrochemical biosensors for monitoring noncommunicable disease biomarkers Biosens. Bioelectron. $130276-292$.

[10] An G, Li S, Wang H, Zhang X, and Yan X 2018 Quasi-D-shaped optical fiber plasmonic refractive index sensor J. Opt.20 035403.

[11] Xie, T, He, Y, Yang, Y, Zhang, H, and Xu, Y 2021 Highly sensitive surface plasmon resonance sensor based on graphene-coated U-shaped fiber Plasmonics 16205 213.

[12] Ghayoor R, and Keshavarz A 2019 Design of Tunable Devices at Terahertz Frequencies Based on Quasi-Photonic Crystals Incorporated with Graphene Commun. Theor. Phys. 711227. 
[13] Jiang W, Gu L, Chen X, and Chen R T 2007 Photonic crystal waveguide modulators for silicon photonics: Device physics and some recent progress Solid. State. Electron. 51 1278-1286.

[14] Bendjelloul R, Bouchemat T, Bouchemat M and Benmerkhi A 2016 New design of T-shaped channel drop filter based on photonic crystal ring resonator J NANOSCI NANOTECHNO. 6 13-17.

[15] Hassan A K S, Mohamed A S, Maghrabi M M, and Rafat N H 2015 Optimal design of one-dimensional photonic crystal filters using minimax optimization approach Appl. Opt. 54 1399-1409.

[16] Lu Y, Liu H, Sun Q, Huang N and Wang Z 2016 Terahertz narrow-band filter based on rectangle photonic crystal J. Mod. Opt. 63 224-230.

[17] Pacholski C 2013 Photonic crystal sensors based on porous silicon Sensors 13 46944713.

[18] Bian L A, Liu P and Li G 2016 Design of tunable devices using one-dimensional Fibonacci photonic crystals incorporating graphene at terahertz frequencies SUPERLATTICE MICROST 98 522-534.

[19] Phan T 2020 Novel coronavirus: From discovery to clinical diagnostics Infect. Genet. Evol. 79104211.

[20] Nishiura H, Linton N M, and Akhmetzhanov A R 2020 Serial interval of novel coronavirus (COVID-19) infections Int. J. Infect. Dis. 93 284-286.

[21] Jiang S, Xia S, Ying T, and Lu L 2020 A novel coronavirus (2019-nCoV) causing pneumonia-associated respiratory syndrome Cell. Mol. Immunol.17 554-554.

[22] Jin X, Lian J S, Hu J H, Gao J, Zheng L, Zhang Y M, ... and Yang Y 2020 Epidemiological, clinical and virological characteristics of 74 cases of coronavirus-infected disease 2019 (COVID-19) with gastrointestinal symptoms Gut 69 1002-1009.

[23] Lu S, Lin J, Zhang Z, Xiao L, Jiang Z, Chen J, ... and Luo S 2021 Alert for nonrespiratory symptoms of coronavirus disease 2019 patients in epidemic period: a case report of familial cluster with three asymptomatic COVID-19 patients $J$. Med. Virol. 93 518-521.

[24] Sahel S, Amri R, Bouaziz L, Gamra D, Lejeune M, Benlahsen M and Bouchriha H 2016 Optical filters using Cantor quasi-periodic one dimensional photonic crystal based on $\mathrm{Si} / \mathrm{SiO} 2$ SUPERLATTICE MICROST 97 429-438. 
[25] Alagdar M, Yousif B, Areed N F and Elzalabani M 2020 Improved the quality factor and sensitivity of a surface plasmon resonance sensor with transition metal dichalcogenide 2D nanomaterials J. Nanoparticle Res. 22 1-13.

[26] Rahman M M, Rana M M, Rahman M S, Anower M S, Mollah M A and Paul A K 2020 Sensitivity enhancement of SPR biosensors employing heterostructure of PtSe2 and 2D materials Opt. Mater. 107110123.

[27] Malitson I H 1965 Interspecimen comparison of the refractive index of fused silica Josa 55 1205-1209.

[28] J Xu 2018 Linear Optical Characterization of Graphene Structure Master's thesis University of Waterloo.

[29] Dai X, Jiang L and Xiang Y 2015 Tunable THz angular/frequency filters in the modified Kretschmann-Raether configuration with the insertion of single layer graphene IEEE Photonics J. 7 1-8.

[30] Ouyang Q, Zeng S, Jiang L, Hong L, Xu G, Dinh X Q ... and Yong K T 2016 Sensitivity enhancement of transition metal dichalcogenides/silicon nanostructure-based surface plasmon resonance biosensor Sci. rep. 6 1-13.

[31] Peterlinz K A, Georgiadis R M, Herne T M, and Tarlov M J 1997 Observation of hybridization and dehybridization of thiol-tethered DNA using two-color surface plasmon resonance spectroscopy J. Am. Chem. Soc. 119 3401-3402.

[32] Prevenslik T 2020 Mystery pulmonary and neurological symptoms by EM radiation from the Coronavirus.

[33] Uddin S M A, Chowdhury S S, and Kabir E 2021 Numerical analysis of a highly sensitive surface plasmon resonance sensor for sars-cov-2 detection Plasmonics $1-13$.

[34] Das C M, Guo Y, Yang G, Kang L, Xu G, Ho H P and Yong K T 2020 Gold Nanorod Assisted Enhanced Plasmonic Detection Scheme of COVID-19 SARS-CoV-2 Spike Protein Adv. Theory Simul. 32000185.

[35] Chowdhury S S, Uddin S M A, and Kabir E 2020 Numerical analysis of sensitivity enhancement of surface plasmon resonance biosensors using a mirrored bilayer structure Photonics Nanostruct PHOTONIC NANOSTRUCT 41100815.

[36] Moznuzzaman M, Islam M R, Hossain M B, and Mehedi I M 2020 Modeling of highly improved SPR sensor for formalin detection Results Phys. 16102874. 
[37] Rahman M S, Anower M S, Bashar L B and Rikta K A 2017 Sensitivity analysis of graphene coated surface plasmon resonance biosensors for biosensing applications Sensing and bio-sensing research 16 41-45. 